\title{
Effects of isoflurane on complex II-associated mitochondrial respiration and reactive oxygen species production: Roles of nitric oxide and mitochondrial $K_{\mathrm{ATP}}$ channels
}

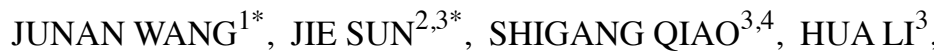 \\ TUANJIE $\mathrm{CHE}^{5}$, CHEN WANG ${ }^{3,4}$ and JIANZHONG $\mathrm{AN}^{3}$
}

\author{
${ }^{1}$ Department of Anesthesiology, Pudong New Area People's Hospital Affiliated to Shanghai University of Medicine \\ and Health Sciences, Shanghai 201299; ${ }^{2}$ Department of Gastroenterology; ${ }^{3}$ Institute of Clinical Medicine Research; \\ ${ }^{4}$ Department of Anesthesiology and Perioperative Medicine; ${ }^{5}$ Laboratory of Precision Medicine and \\ Translational Medicine, The Affiliated Suzhou Science and Technology Town Hospital \\ of Nanjing Medical University, Suzhou, Jiangsu 215153, P.R. China
}

Received March 28, 2019; Accepted August 12, 2019

DOI: $10.3892 / \mathrm{mmr} .2019 .10658$

\begin{abstract}
Volatile anesthetics may protect the heart against ischemia-reperfusion injury via the direct action on mitochondrial complexes and by regulating the production of reactive oxygen species (ROS). Recently, we reported that isoflurane induced the attenuation of mitochondrial respiration caused by complex I substrates. This process was not associated with endogenous production of mitochondrial nitric oxide (NO). In the present study, we investigated the effects of isoflurane on mitochondrial respiration and ROS production using complex II substrates. The detailed mechanism of these effects was explored with regards to NO production and the expression of mitochondrial ATP-dependent $\mathrm{K}^{+}\left(\mathrm{mK}_{\mathrm{ATP}}\right)$ channels. Mitochondria were isolated from the heart of Sprague-Dawley rats. The respiratory rates of mitochondria $(0.5 \mathrm{mg} / \mathrm{ml})$ were measured via polarography at $28^{\circ} \mathrm{C}$ with computer-controlled Clark-type $\mathrm{O}_{2}$ electrodes. The complex II substrate succinate (5 mM) was used; $0.25 \mathrm{mM}$ of isoflurane was administered prior to ADP-initiated state 3 respiration. The mitochondrial membrane potential $\left(\Delta \Psi_{\mathrm{m}}\right)$ was measured under treatment with the substrate succinate, or succinate in the presence of the complex I inhibitor rotenone. The detection was achieved in a cuvette-based spectrophotometer operating at wavelengths of $503 \mathrm{~nm}$ (excitation) $527 \mathrm{~nm}$ (emission) in the presence of $50 \mathrm{nM}$
\end{abstract}

Correspondence to: Professor Jianzhong An, Institute of Clinical Medicine Research, The Affiliated Suzhou Science and Technology Town Hospital of Nanjing Medical University, 1 Lijiang Road, Gaoxin, Suzhou, Jiangsu 215153, P.R. China

E-mail: szkjcyy_ajz@126.com

*Contributed equally

Key words: isoflurane, mitochondria, complex II, respiration, reactive oxygen species of the fluorescent dye rhodamine 123 . The $\mathrm{H}_{2} \mathrm{O}_{2}$ release rates in the mitochondria were measured spectrophotometrically with succinate, or succinate and rotenone using the fluorescent dye Amplex red (12.5-25 $\mu \mathrm{M})$. The results indicated that isoflurane increased the state 3 and 4 respiration rates caused by succinate, which were higher than those noted in the control group in the presence of succinate alone. The NOS inhibitor L-NIO or the NO-sensitive guanylyl cyclase $1 \mathrm{H}-[1,2,4]$ oxadiazolo[4,3-a] quinoxalin-1-one did not inhibit the increase in the respiration rate (state 3 ) induced by isoflurane. The ROS scavengers SPBN and manganese (III) tetrakis (4-benzoic acid) porphyrin chloride inhibited the increase in the respiration rate (state 3 and 4) induced by isoflurane. This effect was not noted for the putative $\mathrm{K}_{\mathrm{ATP}}$ channel blockers 5-hydroxydecanoic acid and glibenclamide. Isoflurane caused a greater decrease in the concentration of $\mathrm{H}_{2} \mathrm{O}_{2}$ during ADP-initiated state 3 respiration, and $\mathrm{L}^{-} \mathrm{N}^{5}$-(1-Iminoethyl)-ornithine did not inhibit this effect. In conclusion, isoflurane was determined to modulate mitochondrial respiration and ROS production caused by the complex II substrate succinate. These effects were independent of endogenous mitochondrial NO generation and mitochondrial $\mathrm{K}_{\mathrm{ATP}}$ channel opening.

\section{Introduction}

Volatile anesthetics are known to reduce myocardial ischemia-reperfusion injury (1-3). Mitochondria exert significant cardioprotective roles (4); the cardioprotective effects caused by pretreatment of volatile anesthetics (anesthetic preconditioning, APC) may be triggered by the induction of reactive oxygen species (ROS) and reactive nitrogen species formation, and by the release of nitric oxide (NO) $(1,5)$. NO exhibits several beneficial roles in myocardial ischemia-reperfusion injury $(1,6,7)$. In addition, isoflurane increases myocardial protection following ischemic post-conditioning via a NO-dependent mechanism (2,3). Mitochondria contain nitric oxide synthase (NOS), an enzyme which produces large amounts of NO and regulates respiratory function (8). NO 
inhibits mitochondrial respiration by binding to the respiratory chain enzyme and regulating the function of mitochondrial ATP-dependent $\mathrm{K}^{+}\left(\mathrm{mK}_{\mathrm{ATP}}\right)$ channels (9). The interaction of $\mathrm{NO}$ with the mitochondrial electron transport chain may serve an important role in APC-induced myocardial protection.

Reversible changes in mitochondrial function caused by volatile anesthetics are associated with APC-induced cardioprotective mechanisms (10-12). Volatile anesthetics are known to affect mitochondrial respiration and electron transport chain (ETC) activity (12-14). Riess et al (12) reported that the administration of sevoflurane in isolated cardiac mitochondria during state 2 respiration resulted in an attenuated state 3 respiration. This attenuation was proposed to be mediated by ROS and was not associated with $\mathrm{mK}_{\mathrm{ATP}}$ channel opening (12). Although it is known that ROS induces the protective effects of APC on ischemia-reperfusion injury (1), the mechanism of its action remains unclear. Volatile anesthetics increase the signals generated by ROS, which is a mandatory step in triggering APC $(15,16)$. The use of ROS scavengers blocks APC-induced cardioprotection (15-17). Collectively, these studies suggested that volatile anesthetics may directly affect mitochondrial complexes and ROS production, thereby regulating mitochondrial respiration and bioenergetics. Recently, we reported that isoflurane induced the attenuation of state 3 respiration (18). This occurred in the presence of the complex I substrates glutamate and malate, and was independent of endogenous mitochondrial NO production (18); however, the effects of volatile anesthetics on mitochondrial respiration and ROS production in the presence of complex II substrates require further investigation. Furthermore, the roles of NO production and $\mathrm{mK}_{\mathrm{ATP}}$ channel function in this process have not been elucidated. In the present study, we aimed to determine the effects of isoflurane on mitochondrial respiration, membrane potential $\left(\Delta \Psi_{\mathrm{m}}\right)$ and ROS production in mitochondria isolated from cardiac cells. The cells were analyzed in the presence of the complex II-associated substrates succinate (Suc) with or without the complex I inhibitor rotenone (Rote). Furthermore, the roles of $\mathrm{mK}_{\text {ATP }}$ channel opening in the effects of isoflurane were also examined.

\section{Materials and methods}

Experimental animals. This experimental study was approved by the Animal Care and Use Committee of the Suzhou Science and Technology Town Hospital. Male Sprague-Dawley rats (weight: $250 \pm 50 \mathrm{~g} ; \mathrm{n}=112$ ), aged 8-10 weeks, were purchased from the Animal Center of Suzhou University and were housed at $25^{\circ} \mathrm{C}$ with $60 \%$ humidity under a $12 \mathrm{~h}$ light-dark cycle. Two rats were housed in each cage and were allowed access to food and water ad libitum. The rats used in the present study underwent humane care in accordance with the National Institute Health Guide for the Care and Use of Laboratory Animals (https:/grants.nih.gov/grants/olaw/Guidefor-the-Care-and-use-of-laboratory-animals.pdf) (2).

Drugs and chemicals. Isoflurane was purchased from Abbot Laboratories. The potent NOS inhibitor, L-N ${ }^{5}$-(1-Iminoethyl)-ornithine (L-NIO) was obtained from Calbiochem. The superoxide dismutase mimetic manganese (III) tetrakis (4-benzoic acid) porphyrin chloride (TBAP) was purchased from OxisResearch. The negatively-charged spin trap N-tert-Butyl-a-(2osulfophenyl)nitrone sodium (SPBN), adenosine-diphosphate (ADP), the selective inhibitor of NO-sensitive guanylyl cyclase $1 \mathrm{H}-[1,2,4]$ oxadiazolo[4,3-a] quinoxalin-1-one (ODQ), the mitochondrial $\mathrm{K}_{\mathrm{ATP}}$ channel blocker 5-hydroxydecanoic acid (5-HD), the nonspecific $\mathrm{K}_{\mathrm{ATP}}$ channel blocker glibenclamide (GLB), the complex II substrate Suc, the complex II inhibitor malonate and the complex I blocker Rote were obtained from Sigma-Aldrich (Merck KGaA). Rhodamine 123 and Amplex red were purchased from Molecular Probes (Thermo Fisher Scientific, Inc.). A total of $10 \mu \mathrm{l}$ of isoflurane was originally dispersed by sonication in $1 \mathrm{ml}$ respiration buffer $(200 \mathrm{mM}$ mannitol, $50 \mathrm{mM}$ sucrose, $5 \mathrm{mM} \mathrm{KH}_{2} \mathrm{PO}_{4}, 1 \mathrm{mM}$ EGTA, 5 mM 3-(n-morpholino)propane sulfonic acid, and $0.1 \%$ bovine serum albumin; $\mathrm{pH} 7.15$, adjusted with potassium hydroxide) and $5 \mu \mathrm{l}$ of this solution was added to the chamber with $500 \mu 1$ of mitochondrial suspension. The concentration of isoflurane was determined by gas chromatography $(0.25 \pm 0.02 \mathrm{mM}$; Schimadzu).

Isolation of mitochondria. The animals were anesthetized with an intraperitoneal injection of pentobarbital sodium (50 $\mathrm{mg} / \mathrm{kg}$ ) prior to decapitation. The heart was quickly removed and placed in an ice-cold isolation buffer containing mannitol (200 mM), sucrose (50 mM), $\mathrm{KH}_{2} \mathrm{PO}_{4}(5 \mathrm{mM})$ EGTA (1 mM), 3-(N-Morpholino)propane sulfonic acid (MOPS; $5 \mathrm{mM}$ ) and $0.1 \%$ bovine serum albumin (Sigma-Aldrich; Merck KGaA), pH 7.3, adjusted by potassium hydroxide. The discarded large blood vessels and auricles were removed of any remaining blood with isolation buffer. The ventricle was cut into small pieces of $1 \mathrm{~mm}^{3}$ in volume. Mitochondrial isolation was carried out at $4^{\circ} \mathrm{C}$ as described previously (18). The total protein concentration was determined by a bicinchoninic protein assay using bovine serum albumin as a standard.

Mitochondrial oxygen $\left(\mathrm{O}_{2}\right)$ consumption. Mitochondrial respiration was measured via polarography at $28^{\circ} \mathrm{C}$ with a computer-controlled Clark-type $\mathrm{O}_{2}$ electrode (Hansatech Instruments Ltd.) (14) in $0.5 \mathrm{ml}$ of respiratory buffer containing $\mathrm{KCl}(130 \mathrm{mM}), \mathrm{K}_{2} \mathrm{PH}_{4}(5 \mathrm{mM})$, MOPS $20(\mathrm{mM})$, EGTA $(2.5 \mathrm{mM}), \mathrm{Na}_{4} \mathrm{Pl}_{2} \mathrm{O}_{7}(0.001 \mathrm{mM})$, EDTA $(1 \mathrm{mM})$ and $0.1 \%$ of bovine serum albumin $(\mathrm{pH} 7.2$, adjusted with potassium hydroxide). The solution was mixed with $0.5 \mathrm{mg}$ of mitochondrial protein per milliliter and with Suc $(5 \mathrm{mM})$ as the complex II substrate (19). State 3 respiration was initiated by addition of ADP $(0.25 \mathrm{mM})$. State 4 respiration was monitored following depletion of ADP (Fig. 1, raw recording data). The representative traces of the respiration indicated a high functional quality and structural integrity of the mitochondria after the isolation procedure, as demonstrated by the respiratory control ratio (RCR, state 3/state 4 ). The RCR was estimated at $9.83 \pm 1.51$ in the presence of Suc. The value of each state was based on the mean of each slope. The RCR was calculated as follows: State 3/state 4 . The data were stored online using the manufacture's software.

Experimental protocols of mitochondrial $\mathrm{O}_{2}$ consumption. To elucidate the mechanism of action of isoflurane on mitochondrial respiration, immediately before each experiment, an aliquot of the concentrated mitochondria was added to 


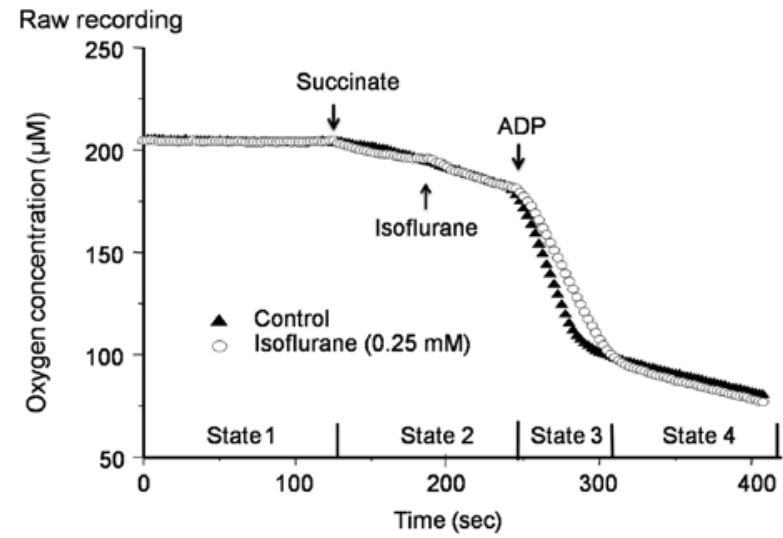

Figure 1. Representative recording traces of mitochondrial respiration. Succinate addition at $2 \mathrm{~min}$ (state 1 transition to state 2). Isoflurane $(0.25 \mathrm{mM})$ was added at state 2 respiration. ADP was added at 4 min (State 2 transition to state 3 ).

respiration buffer at $27^{\circ} \mathrm{C}$. The compound was mixed with Suc in the presence or absence of the complex I inhibitor Rote $(10 \mu \mathrm{M})$ according to the protocols presented in Fig. 2 ( $\mathrm{n}=8$ each protocol). The following compounds were also added: NOS inhibitor L-NIO $(10 \mu \mathrm{M})$, cyclic guanosine monophosphate (cGMP) inhibitor ODQ $(10 \mu \mathrm{m})$, the ROS scavengers TBAP $(10 \mu \mathrm{M})$ and SPBN $(10 \mu \mathrm{M})$, and the $\mathrm{K}_{\text {ATP }}$ channel inhibitors 5-HD $(300 \mu \mathrm{M})$ and GLB $(2 \mu \mathrm{M})$. These experiments used the conventional definition state 2 as the closely analogous steady state in the presence of respiratory substrates and in the absence of additional ADP. The definition state 3 represented the state of maximal phosphorylation after the addition of ADP, while state 4 referred to the state of minimal phosphorylation following depletion of ADP. RCR indicated the degree of the coupling respiration and of the phosphorylation process.

The control group included the substrate Suc alone, or in the presence the complex I (nicotinamide adenine dinucleotide dehydrogenase) inhibitor Rote. These compounds were added at $2 \mathrm{~min}$, while ADP was added at $4 \mathrm{~min}$. Isoflurane was added during state 2 respiration in order to monitor the effect of isoflurane on state 3 and 4 respiration. All other drugs were added prior to isoflurane treatment at $1 \mathrm{~min}$ in order to observe the effects of NO, ROS and $\mathrm{K}_{\mathrm{ATP}}$ channel opening on isoflurane-mediated mitochondrial respiration.

Mitochondrial membrane potential $(\Delta \Psi m)$. Mitochondria $(0.5 \mathrm{mg} / \mathrm{ml})$ were suspended in respiratory buffer. During state 2-4 respiration, $\Delta \Psi \mathrm{m}$ was monitored in the presence of the substrate Suc alone or in the presence of Suc and Rote in a cuvette-based spectrophotometer (Model QM-8, Photon Technology International) operating at excitation and emission wavelengths of 503 and $527 \mathrm{~nm}$, respectively. The detection was conducted in the presence of the fluorescent dye rhodamine $123(50 \mathrm{nM})(20) . \Delta \Psi \mathrm{m}$ was expressed as the percentage of rhodamine 123 fluorescence. The relative mitochondrial fluorescence was subtracted from the value obtained following the addition of the mitochondrial uncoupler, carbonyl cyanide-m-chlorophenylhydrazenone (CCCP, $4 \mu \mathrm{M})$.

Mitochondrial $\mathrm{H}_{2} \mathrm{O}_{2}$ release. During state 2 and 3 respiration, the rates of mitochondrial $\mathrm{H}_{2} \mathrm{O}_{2}$ release in the presence

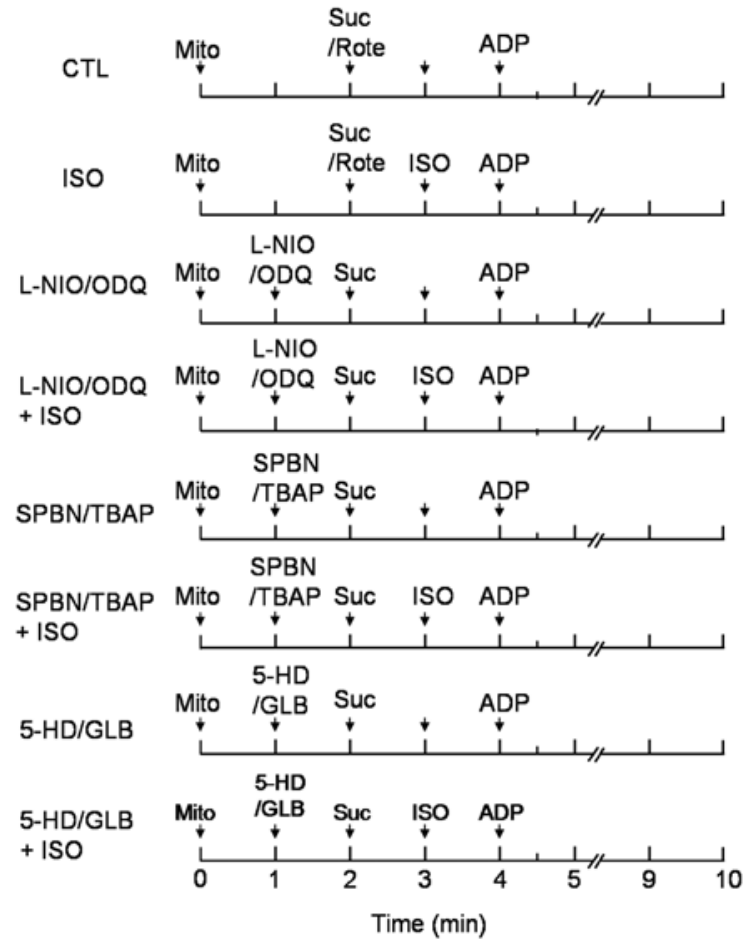

Figure 2. Experimental protocol used for measurement of the mitochondrial respiration state. ISO $(0.25 \mathrm{mM})$ was added at the 2 min period (state 2). The NOS inhibitor L-NIO $(10 \mu \mathrm{M})$, the guanylyl cyclase inhibitor ODQ $(10 \mu \mathrm{m})$, the reactive oxygen species scavengers TBAP $(10 \mu \mathrm{M})$ and SPBN $(10 \mu \mathrm{M})$, and the $\mathrm{K}_{\mathrm{ATP}}$ channel inhibitors 5-HD $(300 \mu \mathrm{M})$ and GLB $(2 \mu \mathrm{M})$ were added at $1 \mathrm{~min}$ (state 1) with or without isoflurane. State 2 respiration was initiated by addition of $5 \mathrm{mM}$ Suc or Suc with the complex I inhibitor rotenone (Rote, $10 \mu \mathrm{M}$ ). State 3 respiration was initiated by addition of $250 \mu \mathrm{M}$ ADP at 4 min. $\mathrm{N}=8$ in each group. 5-HD, 5-hydroxydecanoic acid; CTL, control; GLB, glibenclamide; ISO, isoflurane; Mito, mitochondria; ODQ, 1H-[1,2,4] oxadiazolo[4,3-a]quinoxalin-1-one; Suc, succinate; SPBN, $\mathrm{N}$-tert-Butyl-a-(2osulfophenyl)nitrone sodium; TBAP, tetrakis (4-benzoic acid) porphyrin chloride.

of Suc or Suc and Rote were measured spectrophotometrically (Model QM-8, Photon Technology International) using the fluorescent dye Amplex red $(12.5 \mu \mathrm{M})$ in the presence of $0.1 \mathrm{U} / \mathrm{ml}$ of horseradish peroxidase at $30^{\circ} \mathrm{C}$. Mitochondria $(0.5 \mathrm{mg} / \mathrm{ml})$ were suspended in the respiration buffer, and the reaction was started by the addition of $5 \mathrm{mM}$ succinate $(19,21)$. The excitation and emission wavelengths were set to 530 and $583 \mathrm{~nm}$, respectively. The $\mathrm{H}_{2} \mathrm{O}_{2}$ release rate was expressed as the percentage of baseline $\mathrm{H}_{2} \mathrm{O}_{2}$ release (following addition of the substrate). The baseline $\mathrm{H}_{2} \mathrm{O}_{2}$ levels were calibrated via a standard curve. Specifically three standard curves of photon counts were plotted that were in the range of $10-200 \mathrm{~nm}$ of $\mathrm{H}_{2} \mathrm{O}_{2}$ (Amplex red and horseradish peroxidase were added to the reactants). Each regression was linear and the mean of the standard curves was used $(\mathrm{R}>0.99)$. The control group (dye vehicle) contained $0.1 \%$ dimethyl sulfoxide.

Statistical analysis. All data are expressed as mean \pm standard deviation. Two-way analysis of variance was used to evaluate the differences between the groups. A Student-Newman-Keuls multiple comparison post-hoc test was used to assess the differences within the groups. $\mathrm{P}<0.05$ was considered to indicate a statistically significant difference. 

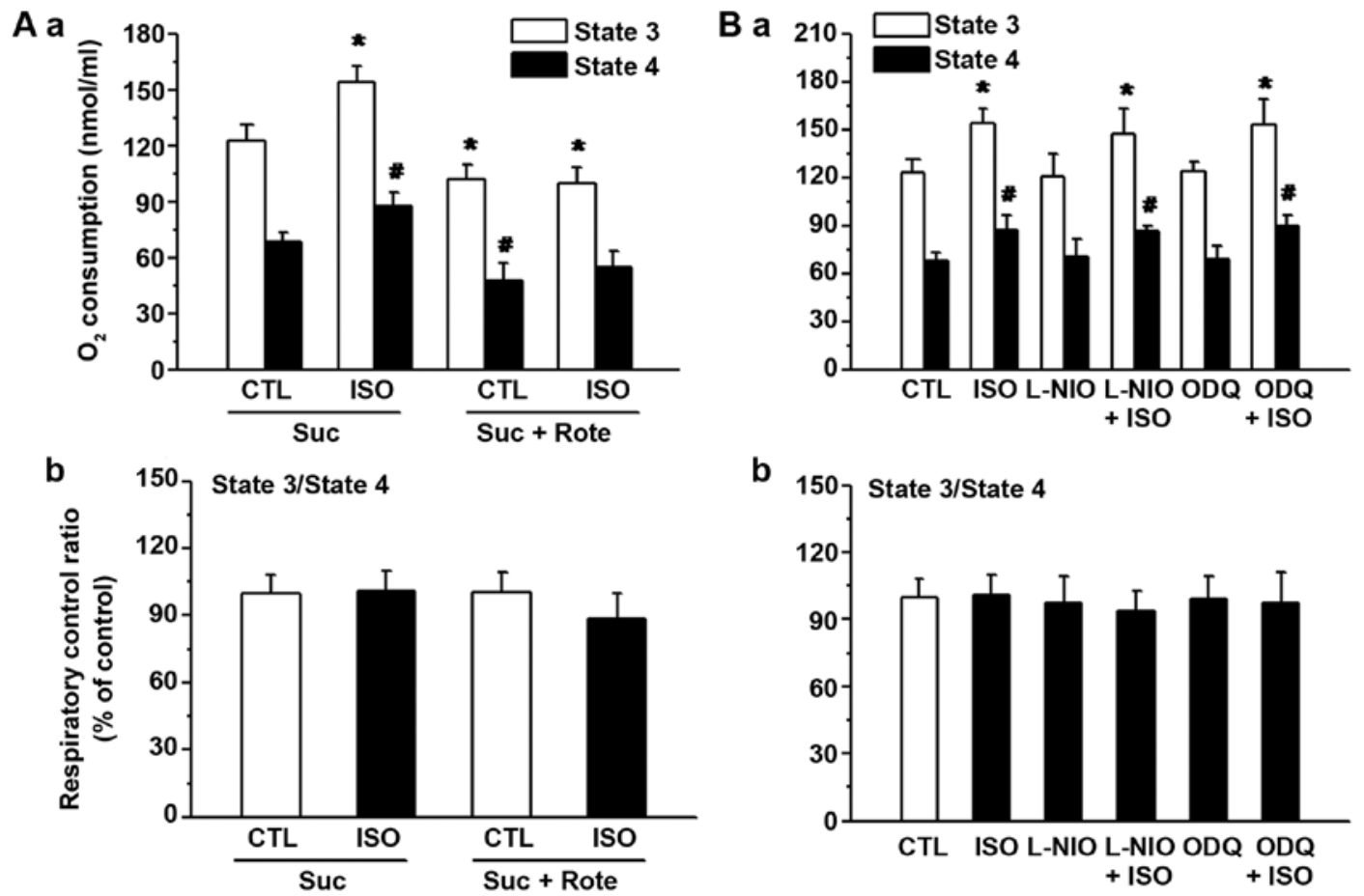

Figure 3. Mitochondrial respiration. Effects of isoflurane $(0.25 \mathrm{mM})$ on the $(\mathrm{A}-\mathrm{a})$ mitochondrial respiration and on (A-b) respiratory control rate prior to the addition of ADP in the presence of succinate with or without Rote. Effects of the nitric oxide synthase inhibitor L-NIO (10 $\mu \mathrm{M})$ or the guanylyl cyclase inhibitor ODQ $(10 \mu \mathrm{m})$ on the (B-a) mitochondrial respiration and (B-b) respiratory control ratio with or without isoflurane in the presence of the complex II substrate Suc. $\mathrm{N}=8$ for each group. ${ }^{*} \mathrm{P}<0.05$ vs. CTL group at state 3 respiration. ${ }^{*} \mathrm{P}<0.05$ vs. CTL group at state 4 respiration. CTL, control; ISO, isoflurane; L-NIO, L-N -(1-Iminoethyl)-ornithine; ODQ, 1H-[1,2,4]oxadiazolo[4,3-a]quinoxalin-1-one; Rote, rotenone; Suc, succinate.

\section{Results}

Mitochondrial respiration. When Suc was used as a substrate, isoflurane significantly increased the state 3 and 4 respiration rates compared with those of the control group (Fig. 3A). Compared with Suc treatment alone, the rate of state 3 respiration was significantly decreased in the control and isoflurane groups in the presence of Suc and Rote. The RCR was markedly reduced in the isoflurane and Rote group; however, this effect was not significantly different compared with that of the control group.

The NOS inhibitor L-NIO or the cyclic cGMP inhibitor ODQ was added prior to the administration of Suc (Fig. 3B). The administration of L-NIO or ODQ did not significantly alter the rates of state 3 and 4 respiration in the L-NIO+ISO group or ODQ+ISO group compared with isoflurane alone group.

The ROS scavengers SPBN or TBAP did not significantly alter the states of mitochondrial respiration compared with that of the control group. Isoflurane significantly increased state 3 and 4 respiration rates; however, SPBN and TBAP inhibited the increase in the isoflurane-induced state 3 and 4 respiration rates in the presence of the substrate Suc (Fig. 4Aa). There was no significance difference between ISO group and SPBN+ISO group or TBAP+ISO group. The putative $\mathrm{K}_{\mathrm{ATP}}$ channel blockers 5-HD and GLB did not inhibit the increase in the isoflurane-induced state 3 and 4 respiration rates in the presence of the substrate Suc (Fig. 4B).

$\Delta \Psi m$. The fluorescence of the membrane potential-sensitive dye rhodamine 123 was measured in order to examine the effects of isoflurane on $\Delta \Psi \mathrm{m}$. The data are presented as the percentage of maximal depolarization by carbonyl cyanide- $m$-chlorophenylhydrazenone (Fig. 5). Administration of isoflurane at state 2 respiration caused no effects on the $\Delta \Psi \mathrm{m}$ noted at state 3 and 4 respiration in the presence of Suc. However, depolarization was significantly promoted by isoflurane at state 3 respiration in the presence of Suc and Rote compared with the control; L-NIO did not significantly alter the effects of isoflurane (Fig. 5A). Of note, significant depolarization of $\Delta \Psi \mathrm{m}$ at state 4 respiration was detected in the Suc and Rote group compared with the control; L-NIO did not notably inhibit depolarization induced by isoflurane (Fig. 5B).

Mitochondrial ROS generation. The baseline levels of mitochondrial $\mathrm{H}_{2} \mathrm{O}_{2}$ release at state 2 respiration were significantly higher in the Suc group compared with the Suc+Rote group. Isoflurane induced a significant decrease in the concentration of $\mathrm{H}_{2} \mathrm{O}_{2}$ compared with the control, while L-NIO markedly inhibited the isoflurane-induced decrease in $\mathrm{H}_{2} \mathrm{O}_{2}$ concentration levels (Fig. 6A). A significant increase in $\mathrm{H}_{2} \mathrm{O}_{2}$ release was observed compared with the control in response to isoflurane with Suc and Rote; this effect was not inhibited by L-NIO (Fig. 6B). Isoflurane and L-NIO did not significantly alter $\mathrm{H}_{2} \mathrm{O}_{2}$ release rates during ADP-initiated state 3 respiration in the Suc or Suc+Rote groups (Fig. 6B).

\section{Discussion}

We recently reported that isoflurane exerts specific effects on different states of mitochondrial respiration during the oxidation of complex I substrates, which may not depend on endogenous mitochondrial NO (18). The results of the current 

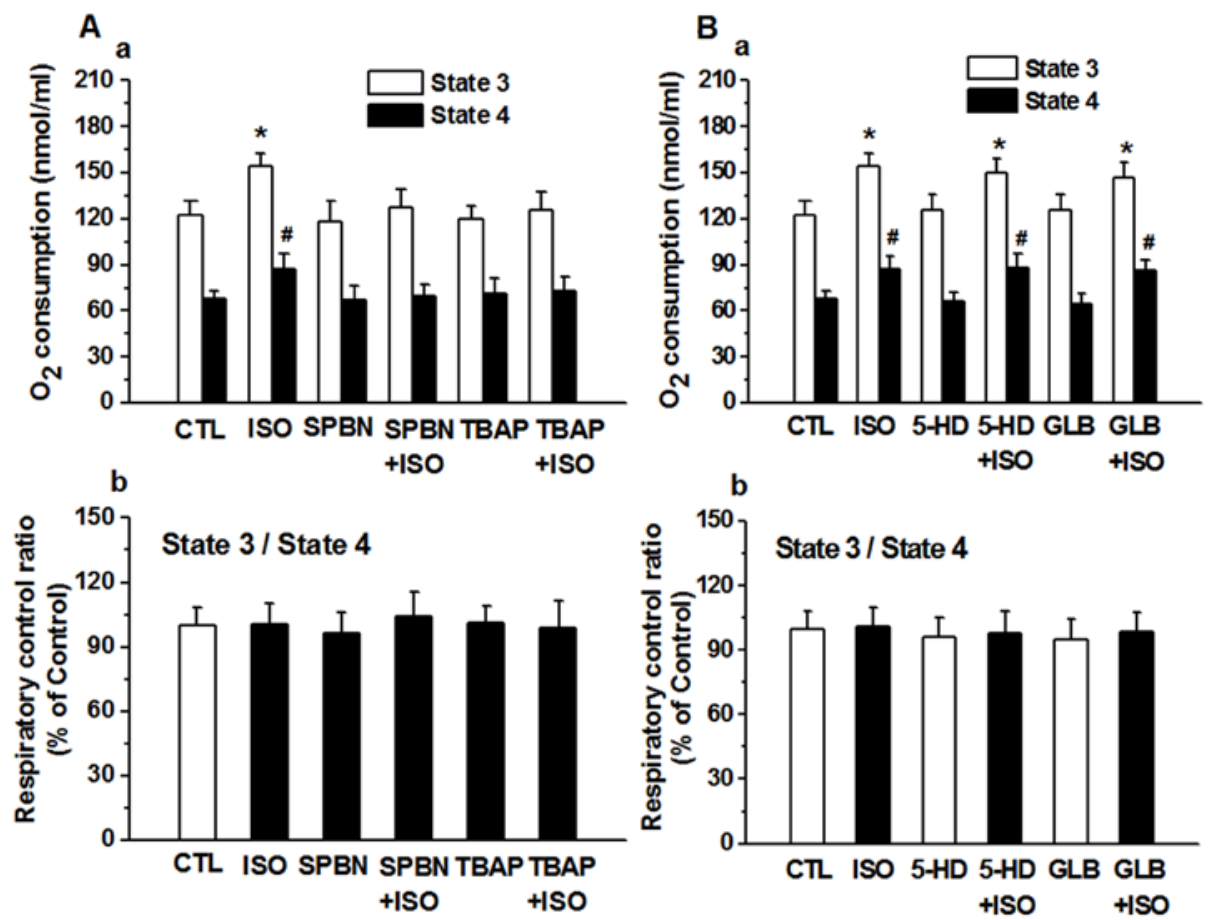

Figure 4. Effects of reactive oxygen species scavengers (A) TBAP $(10 \mu \mathrm{M})$ and SPBN (10 $\mu \mathrm{M})$ on (a) the mitochondrial respiration and on (b) the respiratory control ratio with or without isoflurane in the presence of the complex II substrate succinate. Effects of KATP channel inhibitors (B) 5-HD (300 $\mu \mathrm{M})$ and GLB $(2 \mu \mathrm{M})$ on the (a) mitochondrial respiration and on (b) the respiratory control ratio with or without isoflurane in the presence of the complex II substrate succinate. $\mathrm{N}=8$ for each group. ${ }^{*} \mathrm{P}<0.05$ vs. CTL group at state 3 respiration. ${ }^{*} \mathrm{P}<0.05$ vs. CTL group at state 4 respiration. 5 -HD, 5 -hydroxydecanoic acid; CTL, control; GLB, glibenclamide; ISO, isoflurane; SPBN, N-tert-Butyl-a-(2osulfophenyl)nitrone sodium; TBAP, tetrakis (4-benzoic acid) porphyrin chloride.

study supported previous findings in which: i) Isoflurane increased mitochondrial state 3 respiration in isolated intact mitochondria in the presence of the complex II substrate Suc; ii) this effect of isoflurane was inhibited by Rote; iii) The NOS inhibitor L-NIO or the NO-sensitive guanylyl cyclase ODQ did not inhibit the increase in the respiration rate (state 3 ) caused by isoflurane in the presence of Suc; iv) the ROS scavengers SPBN and TBAP inhibited the increase in the respiration rate (states 3 and 4) caused by isoflurane in the presence of Suc. This effect was not noted by the putative $\mathrm{K}_{\mathrm{ATP}}$ channel blockers 5-HD and GLB; v) isoflurane did not alter state $3 \Delta \Psi_{\mathrm{m}}$ depolarization in the presence of Suc, whereas this peak depolarization was decreased in the presence of Suc and Rote; and vi) isoflurane reduced the concentration of $\mathrm{H}_{2} \mathrm{O}_{2}$ during ADP-initiated state 3 respiration; this effect was not inhibited by L-NIO.

Volatile anesthetics have been reported to protect the myocardium from infrared radiation damage by regulating mitochondrial respiratory function $(18,19,20,22)$. Isoflurane attenuated state 3 respiration and reduced the RCR during the oxidation of the complex I-chain substrate glutamate/malic acid (18). In the present study, isoflurane significantly enhanced state 3 respiration in the presence of Suc alone. This result is consistent with the findings of Hirata et al (19). The analysis of mitochondrial respiration in the presence of Suc alone indicated a higher rate of ROS production, mainly due to the reverse transport of electrons into complex I (23). Agarwal et al (20) demonstrated that isoflurane inhibited the $\mathrm{O}_{2}$ consumption of complex I-chain substrates, but not of complex-II linked substrates (20). In the current study, the results revealed that Suc combined with Rote exhibited no difference in the state 3 respiration rate or the RCR between the control and the isoflurane-treated groups, consistent with the aforementioned study (20). When Suc was used without Rote, isoflurane increased $\mathrm{O}_{2}$ consumption. These results suggested that complex I was the target of isoflurane. The Krebs cycle causes the conversion of Suc to fumarate and malate, and subsequently to oxaloacetate (24). This occurs in the absence of Rote; oxaloacetate causes a decrease in Suc oxidation by inhibiting complex II (19). When complex I activity is inhibited, NADH is oxidized, thereby lowering the levels of $\mathrm{NAD}^{+}$, which in turn disrupts the oxidation of malate to oxaloacetate (25). Therefore, isoflurane may inhibit complex I by attenuating the formation of oxaloacetate, thereby increasing the complex II-associated respiration.

In the presence of the complex I substrates pyruvate and malate, the ROS scavengers TBAP (simulated superoxide dismutase) and SPBN (free radical spin trap) reduce the effects of sevoflurane on state 3 respiration. The application of Suc in the presence of Rote eliminates the effects of the two ROS scavengers on the sevoflurane-induced attenuation of state 3 respiration (18). In the present study isoflurane induced an increase in the state 3 respiration rate with the complex II substrate Suc (in the absence of Rote), whereas TBAP and SPBN (ROS scavengers) inhibited the effects of isoflurane. This suggested that complex I function was promoted by superoxide formation in the absence of Rote during isoflurane exposure, whereas the effects of isoflurane were inhibited in the presence of the scavengers TBAP and SPBN. The results are in agreement with the study by Riess et al (12) in which a positive feedback mechanism of ROS formation was noted in complex III. This feedback effect attenuated the function of complex I, thereby producing additional ROS. 

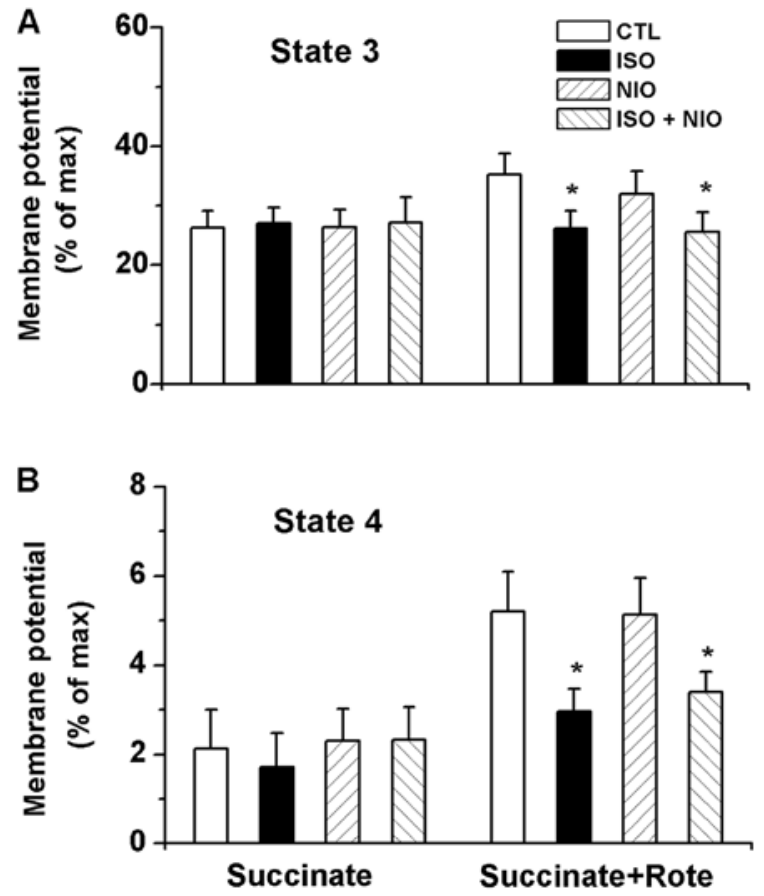

Figure 5. Summarized data for the effects of isoflurane on the mitochondrial membrane potential. (A) State 3 and (B) state 4 respiration in the presence of the complex II substrate succinate with or without rotenone. $\mathrm{N}=8$ for each group. ${ }^{*} \mathrm{P}<0.05$ vs. CTL group. CTL, control; ISO, isoflurane; NIO, L-N5-(1-Iminoethyl)-ornithine; Rote, rotenone.
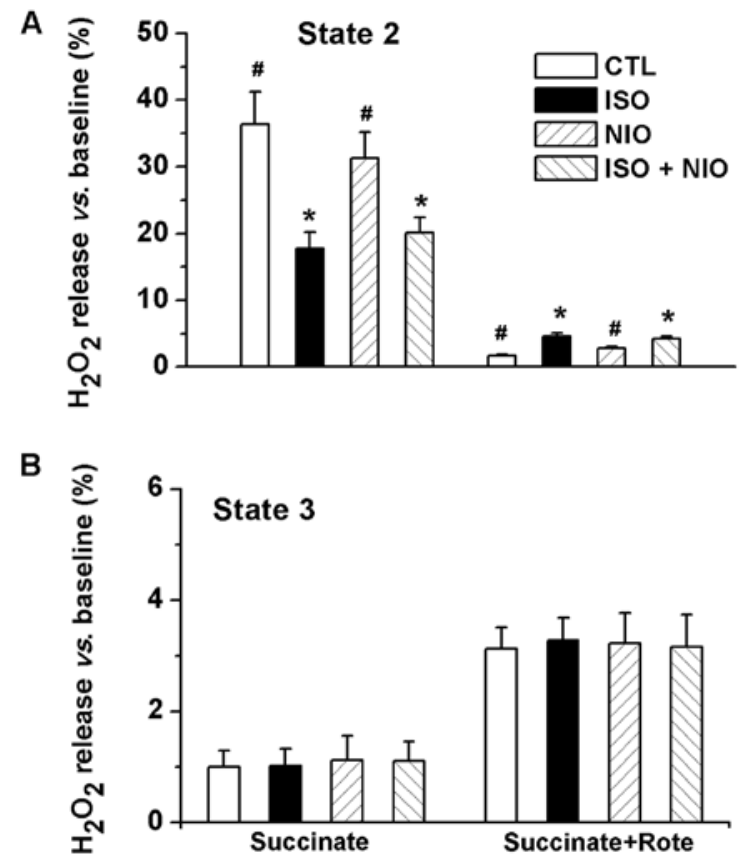

Figure 6. Summarized data for the effects of isoflurane on the $\mathrm{H}_{2} \mathrm{O}_{2}$ release at (A) state 2 and (B) at state 3 respiration in the presence of the complex II substrate succinate with or without rotenone. ${ }^{*} \mathrm{P}<0.05$ vs. corresponding CTL group. $\mathrm{N}=8$ for each group. ${ }^{\#} \mathrm{P}<0.05$ vs. ISO group under Suc or Suc+Rote treatment. CTL, control; ISO, isoflurane; NIO, L-N ${ }^{5}-(1-$ Iminoethyl)ornithine; Rote, rotenone.

The opening of the $\mathrm{mK}_{\mathrm{ATP}}$ channel increases the production of ROS in the mitochondria isolated from heart and liver, while ROS is produced in the electron transport chain and notably BY complex I (26). However, Riess et al (12) reported that complex I-associated attenuation of mitochondrial respiration by the volatile anesthetic sevoflurane was mediated by ROS and was independent of $\mathrm{mK}_{\mathrm{ATP}}$ channel opening. Volatile anesthetics increase ROS formation via complex I and/or complex III by affecting the reversible attenuation of mitochondrial electron transport (12). ROS can affect mitochondrial respiration by acting on downstream effectors, such as the $\mathrm{mK}_{\text {ATP }}$ channel, which is normally closed at physiological ATP levels (27). In agreement with the study by Riess et al (12), the results of the present study indicated that the putative $\mathrm{K}_{\text {ATP }}$ channel blockers 5-HD and GLB did not inhibit isoflurane-induced state 3 and 4 respiration increase in the presence of Suc. Therefore, the involvement of $\mathrm{K}_{\mathrm{ATP}}$ channel or other potassium channel opening with regards to the isoflurane-induced mitochondrial respiratory state can be ruled out.

NO is produced by mitochondrial NOS, and regulates mitochondrial $\mathrm{O}_{2}$ consumption and transmembrane potential (28). The reaction of NO with superoxide anion produces peroxynitrite, which induces oxidation and/or nitrosation by irreversibly altering susceptible targets in the mitochondria (28). The activation of mitochondrial NOS by the volatile anesthetic isoflurane produces NO, which affects respiration. Our previous study demonstrated that the potent NOS inhibitor L-NIO did not alter the effects of isoflurane on mitochondrial respiration and did not affect normal mitochondrial respiration in the presence of complex I substrates (18). Guanylate cyclase (GC) converts GTP to cGMP and catalyzes the synthesis of cGMP. NO activates soluble GC (sGC) and promotes cGMP synthesis; sGC is inhibited by ODQ (29). In the current study, the NOS inhibitor L-NIO or the sGC inhibitor ODQ were added prior to the administration of isoflurane in the presence of complex II substrates. These compounds demonstrated the same results as those noted in the presence of the complex I substrates. This finding further confirmed that the production of endogenous mitochondrial NO did not influence the effects of isoflurane on mitochondrial respiration.

The high production of ROS relies on high internal $\Delta \Psi \mathrm{m}$, which is sensitive to complex I inhibition. Therefore, Rote could prevent ROS production under reverse electron flow conditions $(30,31)$. In the presence of high proton motive force, electrons are passed to NAD until the electron pool is completely depleted and NAD is reduced to NADH (31). Subsequently, all upstream redox centers are fully reduced and semiquinone transfers its unpaired electrons to $\mathrm{O}_{2}$ (31). It is known that additional ROS are produced under the FADH2-ligating substrates during mitochondrial respiration $(30,32)$. ROS production in the presence of Suc occurs due to the reverse electron flow into the complex I of ETC, which is mainly dependent on the high $\Delta \Psi_{\mathrm{m}}(33,34)$. The results of the present study supported the aforementioned observations. A mitochondrial uncoupler was used, namely CCCP, which allowed protons to re-enter to the inner mitochondrial membrane and inhibit the production of $\mathrm{H}_{2} \mathrm{O}_{2}$, which led to the collapse of $\Delta \Psi \mathrm{m}$. Any decrease in $\Delta \Psi \mathrm{m}$ would abolish ROS generation at this site. In addition, reverse electron flow-induced ROS production can be highly attenuated by the complex I blocker Rote and ADP. This rationale is partially in agreement with the present findings, as the data indicated that the depolarization of $\Delta \Psi \mathrm{m}$ was reduced by isoflurane and L-NIO in the presence of Suc and Rote. The 
decrease in mitochondrial damage was caused by the block of the electron flow from complex I during myocardial ischemia-reperfusion, which in turn occurred due to the reduction in ROS production (35).

The production of ROS in the mitochondria is initiated in the form of superoxide, by transferring electrons directly to $\mathrm{O}_{2}$ (electron leakage) and converting them to $\mathrm{H}_{2} \mathrm{O}_{2}$ via superoxide dismutase (28). A previous study has shown that isoflurane reduced ROS production in intact mitochondria in the presence of Suc, whereas in the presence of Rote, opposing effects were noted (ROS increase) (19). The results of the present study further indicated that isoflurane significantly decreased the baseline levels of $\mathrm{H}_{2} \mathrm{O}_{2}$ release in the Suc group during state 2 respiration, whereas it increased the baseline levels of $\mathrm{H}_{2} \mathrm{O}_{2}$ release during state 3 respiration in the Suc and Rote group. This effect was possibly mediated via reverse electron flow. Endogenous mitochondrial NO production was not involved in the decreased $\mathrm{H}_{2} \mathrm{O}_{2}$ concentration levels induced by isoflurane, since the NOS inhibitor L-NIO did not affect the levels of $\mathrm{H}_{2} \mathrm{O}_{2}$ release. Due to the significant increase in the concentration of Suc during hypoxia, the effect of isoflurane may be particularly relevant during myocardial ischemia (36). Therefore, isoflurane could reduce ROS production and may contribute to APC-induced cardioprotective mechanisms. It has been reported in isolated cardiac mitochondria that ROS serve different roles in sevoflurane-induced ETC attenuation (12).

The present study indicated that isoflurane increased mitochondrial state 3 respiration in isolated cardiac mitochondria in the presence of the complex II substrate and further confirmed that the effect of isoflurane on the mitochondrial respiration was not associated with endogenous mitochondrial NO production. Isoflurane decreased the depolarization of $\Delta \Psi \mathrm{m}$, thus reducing ROS production caused by reverse electron flow; however, this effect was determined to be independent of $\mathrm{mK}_{\text {ATP }}$ channel opening. Altered mitochondrial function is induced by volatile anesthetics and attenuates ischemia/reperfusion injury (20). This provides an important component in deciphering the complexity of cardiac protection $(12,20)$. The findings reported in the present study suggest a pivotal role for isoflurane in regulating mitochondrial respiration and ROS production, and provide useful information of the possible synergistic action of volatile anesthetics at multiple sites of the mitochondria. These synergistic effects may be the basis of the cardioprotective effects induced by such compounds. Furthermore, the effects of volatile anesthetics on mitochondrial function under pathological conditions, and those involved in induced cardiac protection require further investigation.

\section{Acknowledgements}

Not applicable.

\section{Funding}

The present study was supported by grants (grant no. SS201756 to Dr An and grant no. SS201613 to Dr Wang) from the Suzhou Science and Technology Development Plan. In addition, grants from the National Science and Technology Development Plan (grant no. NSFC 81703501 to Dr Qiao), and from the
Suzhou New District Science and Technology Project (grant no. $2017 Z 004$ to Dr An and grant no. 2017Q003 to Lei Hong).

\section{Authors' contributions}

JW, JS, TC, CW and JA participated in conceiving and designing the experiments; JW, JS, HL and SQ conducted the experiments; JW, JS, TC, CW and JA were involved in the interpretation of the studies, the analysis of the data and reviewed of the manuscript; JW, JS and JA prepared the manuscript draft. All authors read and approved the final version of the manuscript.

\section{Availability of data and materials}

The datasets used and/or analyzed during the present study are available from the corresponding author on reasonable request.

\section{Ethics approval and consent to participate}

The present study was approved by the Animal Care and Use Committee of the Suzhou Science and Technology Town Hospital.

\section{Patient consent for publication}

Not applicable.

\section{Competing interests}

The authors declare that they have no competing interests.

\section{References}

1. Swyers T, Redford D and Larson DF: Volatile anesthetic-induced preconditioning. Perfusion 29: 10-15, 2014.

2. Ge ZD, Pravdic D, Bienengraeber M, Pratt PF Jr, Auchampach JA, Gross GJ, Kersten JR and Warltier DC: Isoflurane postconditioning protects against reperfusion injury by preventing mitochondrial permeability transition by an endothelial nitric oxide synthase-dependent mechanism. Anesthesiology 112: 73-85, 2010.

3. Cao J, Xie H, Sun Y, Zhu J, Ying M, Qiao S, Shao Q, Wu H and Wang C: Sevoflurane post-conditioning reduces rat myocardial ischemia reperfusion injury through an increase in NOS and a decrease in phopshorylated NHE1 levels. Int J Mol Med 36: 1529-1537, 2015.

4. Camara AK, Bienengraeber M and Stowe DF: Mitochondrial approaches to protect against cardiac ischemia and reperfusion injury. Front Physiol 2: 13, 2011.

5. Li H and Lang XE: Protein kinase C signaling pathway involvement in cardioprotection during isoflurane pretreatment. Mol Med Rep 11: 2683-2688, 2015.

6. Bolli R: Cardioprotective function of inducible nitric oxide synthase and role of nitric oxide in myocardial ischemia and preconditioning: An overview of a decade of research. J Mol Cell Cardiol 33: 1897-1918, 2001.

7. Chiari PC, Bienengraeber MW, Weihrauch D, Krolikowski JG, Kersten JR, Warltier DC and Pagel PS: Role of endothelial nitric oxide synthase as a trigger and mediator of isoflurane-induced delayed preconditioning in rabbit myocardium. Anesthesiology 103: 74-83, 2005.

8. Brown GC: Nitric oxide and mitochondrial respiration. Biochim Biophys Acta 1411: 351-369, 1999.

9. Shiva S, Oh JY,Landar AL, Ulasova E, Venkatraman A, Bailey SM and Darley-Usmar VM: Nitroxia: The pathological consequence of dysfunction in the nitric oxide-cytochrome c oxidase signaling pathway. Free Radic Biol Med 38: 297-306, 2005. 
10. An J, Camara AK, Riess ML, Rhodes SS, Varadarajan SG and Stowe DF: Improved mitochondrial bioenergetics by anesthetic preconditioning during and after 2 hours of 27 degrees $\mathrm{C}$ ischemia in isolated hearts. J Cardiovasc Pharmacol 46: 280-287, 2005.

11. An J, Camara AK, Rhodes SS, Riess ML and Stowe DF: Warm ischemic preconditioning improves mitochondrial redox balance during and after mild hypothermic ischemia in guinea pig isolated hearts. Am J Physiol Heart Circ Physiol 288: H2620-H2627, 2005.

12. Riess ML, Eells JT, Kevin LG, Camara AK, Henry MM and Stowe DF: Attenuation of mitochondrial respiration by sevoflurane in isolated cardiac mitochondria is mediated in part by reactive oxygen species. Anesthesiology 100: 498-505, 2004.

13. Hanley PJ, Ray J, Brandt U and Daut J: Halothane, isoflurane and sevoflurane inhibit NADH: Ubiquinone oxidoreductase (complex I) of cardiac mitochondria. J Physiol 544: 687-693, 2002.

14. Ljubkovic M, Mio Y, Marinovic J, Stadnicka A, Warltier DC, Bosnjak ZJ and Bienengraeber M: Isoflurane preconditioning uncouples mitochondria and protects against hypoxia-reoxygenation. Am J Physiol Cell Physiol 292: C1583-C1590, 2007.

15. Tanaka K, Weihrauch D, Kehl F, Ludwig LM, LaDisa JF Jr, Kersten JR, Pagel PS and Warltier DC: Mechanism of preconditioning by isoflurane in rabbits: A direct role for reactive oxygen species. Anesthesiology 97: 1485-1490, 2002.

16. Kevin LG, Novalija E, Riess ML, Camara AK, Rhodes SS and Stowe DF: Sevoflurane exposure generates superoxide but leads to decreased superoxide during ischemia and reperfusion in isolated hearts. Anesth Analg 96: 949-955, 2003.

17. Novalija E, Kevin LG, Eells JT, Henry MM and Stowe DF: Anesthetic preconditioning improves adenosine triphosphate synthesis and reduces reactive oxygen species formation in mitochondria after ischemia by a redox dependent mechanism. Anesthesiology 98: 1155-1163, 2003

18. Xu F, Qiao S, Li H, Deng Y, Wang C and An J: The effect of mitochondrial complex I-linked respiration by isoflurane is independent of mitochondrial nitric oxide production. Cardiorenal Med 28: 113-122, 2018.

19. Hirata N, Shim YH, Pravdic D, Lohr NL, Pratt PF Jr, Weihrauch D, Kersten JR, Warltier DC, Bosnjak ZJ and Bienengraeber M: Isoflurane differentially modulates mitochondrial reactive oxygen species production via forward versus reverse electron transport flow: Implications for preconditioning. Anesthesiology 115: 531-540, 2011.

20. Agarwal B, Dash RK, Stowe DF, Bosnjak ZJ and Camara AK Isoflurane modulates cardiac mitochondrial bioenergetics by selectively attenuating respiratory complexes. Biochim Biophys Acta 1837: 354-365, 2014

21. Heinen A, Camara AK, Aldakkak M, Rhodes SS, Riess ML and Stowe DF: Mitochondrial Ca2+-induced $\mathrm{K}+$ influx increases respiration and enhances ROS production while maintaining membrane potential. Am J Physiol Cell Physiol 292: C148-C156, 2007.
22. Harisseh R, Chiari P, Villedieu C, Sueur P, Abrial M, Fellahi JL, Ovize $M$ and Gharib A: Cyclophilin D modulates the cardiac mitochondrial target of isoflurane, sevoflurane, and desflurane. J Cardiovasc Pharmacol 69: 326-334, 2017.

23. Saborido A, Soblechero L and Megias A: Isolated respiring heart mitochondria release reactive oxygen species in states 4 and 3 . Free Radic Res 39: 921-931, 2005.

24. Ackrell BA, Kearney EB and Mayr M: Role $3 \mathrm{f}$ oxalacetate in the regulation of mammalian succinate dehydrogenase. $\mathrm{J}$ boil Chem 249: 2021-2027, 1974.

25. Esteitie N, Hinttala R, Wibom R, Nilsson H, Hance N, Naess K, Tear-Fahnehjelm K, von Döbeln U, Majamaa K and Larsson NG: Secondary metabolic effects in complex I deficiency. Ann Neurol 58: 544-552, 2005

26. Andrukhiv A, Costa AD, West IC and Garlid KD: Opening mitoKATP increases superoxide generation from complex I of the electron transport chain. Am J Physiol Heart Circ Physiol 291: H2067-H2074, 2006.

27. Zaugg M, Lucchinetti E, Spahn DR, Pasch T and Schaub MC: Volatile anesthetics mimic cardiac preconditioning by priming the activation of mitochondrial K(ATP) channels via multiple signaling pathways. Anesthesiology 97: 4-14, 2002.

28. Stowe DF and Camara AK: Mitochondrial reactive oxygen species production in excitable cells: Modulators of mitochondrial and cell function. Antioxid Redox Signal 11: 1373-1414, 2009.

29. Denninger JW and Marletta MA: Guanylate cyclase and the .NO/cGMP signaling pathway. Biochim Biophys Acta 1411: 334-350, 1999.

30. Loschen G, Flohe $L$ and Chance B: Respiratory chain linked H(2) $\mathrm{O}(2)$ production in pigeon heart mitochondria. FEBS Lett 18: 261-264, 1971.

31. Lambert AJ and Brand MD: Inhibitors of the quinone-binding site allow rapid superoxide production from mitochondrial NADH: Ubiquinone oxidoreductase (complex I). J Biol Chem 279: 39414-39420, 2004

32. Turrens JF and Boveris A: Generation of superoxide anion by the NADH dehydrogenase of bovine heart mitochondria. Biochem J 1191: 421-427, 1980 .

33. Liu Y, Fiskum G and Schubert D: Generation of reactive oxygen species by the mitochondrial electron transport chain. J Neurochem 80: 780-787, 2002.

34. Heinen A, Aldakkak M, Stowe DF, Rhodes SS, Riess ML Varadarajan SG and Camara AK: Reverse electron flow-induced ROS production is attenuated by activation of mitochondrial Ca2+-sensitive K+ channels. Am J Physiol Heart Circ Physiol 293: H1400-H1407, 2007.

35. Chen Q, Camara AK, Stowe DF, Hoppel CL and Lesnefsky EJ: Modulation of electron transport protects cardiac mitochondria and decreases myocardial injury during ischemia and reperfusion. Am J Physiol Cell Physiol 292: C137-C147, 2007.

36. Hoyer S and Krier C: Ischemia and aging brain. Studies on glucose and energy metabolism in rat cerebral cortex. Neurobiol Aging 7: 23-29, 1986. 